\title{
Principios activos de bajo período de carencia para el control de plagas y enfermedades en verduras de hoja.
}

\author{
Pinto Ruiz, Gabriel A.; Tarragó, José R.; Álvarez, Roberto E. \\ Cátedra de Terapéutica Vegetal - Facultad de Ciencias Agrarias - Universidad Nacional del Nordeste. \\ E-mail: gabrielpintoruiz@gmail.com
}

\begin{abstract}
RESUMEN
La producción de hortalizas de hoja es un sistema intensivo, cuyos productos son comercializados en fresco por lo que deben cumplir condiciones de inocuidad para los consumidores. La utilización de métodos de control químico en estos sistemas debe considerar el cumplimiento de uso de productos fitosanitarios autorizados, de bajo impacto ambiental y de los días de carencia para cada situación, producto y dosis. Considerando lo anteriormente expuesto se realizó una recopilación bibliográfica de los productos autorizados para el control de plagas en cultivos hortícolas de hojas a los fines de facilitar a los asesores técnicos la toma de decisiones en el momento de realizar una recomendación de control químico. Esta guía está compuesta por listas de principios activos registrados con hasta 8 días de carencia y sus valores de EIQ ecológico como herramienta a la hora de seleccionar un principio activo. En el siguiente trabajo se presenta una lista de principios activos permitidos para usar en el control de plagas en los cultivos de acelga, espinaca, rúcula, perejil, lechuga, repollo, apio y achicoria, como así también información importante a la hora de realizar una prescripción de un tratamiento fitosanitario. La utilización adecuada de esta información contribuiría a una producción de hortalizas inocuas para el consumo y con menor impacto ambiental posible.
\end{abstract}

Palabras Clave: Verduras de hoja; Impacto ambiental; Fitosanitarios

\section{INTRODUCCIÓN}

La producción de verduras de hoja, como acelga, lechuga y rúcula es estacional, ya que las mismas se cultivan en ciertos períodos del año, su ciclo es corto y la cosecha se realiza en forma escalonada durante un corto tiempo. En este sentido se puede considerar que un cultivo de acelga tiene un tiempo a cosecha de 60 a 80 días en siembras de primavera, la espinaca posee un período de cultivo de 40 a 45 días en épocas primaverales y el perejil tiene un lapso de 10 a 15 días entre cortes de cosecha de hojas según las temperaturas (Goites, 2008). En el momento de tomar una decisión de control químico se deben considerar varios aspectos como ser: emplear productos registrados para el cultivo en cuestión, que sea eficiente para la plaga que queremos controlar y que el tiempo de carencia posibilite reiniciar la cosecha sin que esto produzca pérdidas y peligros de intoxicación para los operarios y/o consumidores. El tiempo de carencia se define como "la cantidad de días que deben transcurrir entre la última aplicación de un producto fitosanitario y la cosecha del cultivo o pastoreo de animales" (CASAFE, 2015). Este tiempo legalmente establecido, se expresa usualmente en número de días y es estudiado para cada producto, cultivo y dosis. Nos asegura llegar al momento de cosecha con un nivel de residuo inferior a la ingesta diaria admitida y por lo tanto la inocuidad

Recibido: 18/09/2017. Aceptado: 16/02 del alimento para el consumo (Pacheco y Barbona, 2017). Un fácil acceso a esta información es crucial a la hora de utilizar un producto fitosanitario y aunque en algunos casos los días de carencia se encuentran en los marbetes o guías fitosanitarias, no siempre se los halla con facilidad ya que esta información se encuentra en resoluciones de SENASA que no son conocidas por el productor e imposibilita el acceso a esta información. En muchos cultivos regionales o en los cuales el área cultivada es baja no existen laboratorios que quieran hacer los registros de sus productos y en estos casos SENASA reconoce una extensión de uso para el control de una plaga, y los tiempos de carencia y otras especificaciones solo están en las resoluciones del organismo.

La mayoría de los sistemas agrícolas actuales utiliza el control químico para una optimización de la productividad y por ende éste debe ser manejado con precaución a fin de minimizar el efecto sobre el ambiente, sobre los trabajadores y los consumidores. En este sentido, toda aplicación de un agroquímico conlleva un impacto ambiental que dependerá de las propiedades del producto fitosanitario (formulación, toxicidad, persistencia, volatilidad, solubilidad en agua y adsorción al suelo), como así también de las características del suelo, del método de aplicación, el clima y otros factores (Arregui et al., 2009; Reyes et al., 2010; Arregui et al., 2013). 
En la actualidad existen varios indicadores que permiten estimar el impacto ambiental que genera un fitosanitario; y a su vez también es posible cuantificar el impacto que generan las distintas aplicaciones de agroquímicos en un ciclo productivo. El EIQ (Coeficiente de Impacto Ambiental) es una medida del impacto ambiental que produce el uso de un agroquímico en un determinado ambiente dosis y concentración (Kovach et al., 1992). El modelo del EIQ utiliza una ecuación basada en tres componentes principales de los sistemas de producción agrícola: 1) componente de trabajadores agrícolas, 2) componente de consumidor, 3) componente ecológico y valora el impacto con un único valor cuantitativo y adimensional.

En el cálculo del EIQ se consideran todos los aspectos relacionados a cada principio activo como: $\mathrm{DT}=$ toxicidad dermal; $\mathrm{C}=$ toxicidad crónica; $\mathrm{SY}=$ sistematicidad; $\mathrm{F}=$ toxicidad para peces; $\mathrm{D}=$ toxicidad para aves; $\mathrm{S}=$ vida media en el suelo; $\mathrm{L}=$ potencial de lixiviación; $\mathrm{R}=$ perdida potencial por superficie; $\mathrm{Z}=$ toxicidad para las abejas; $\mathrm{B}=$ toxicidad para artrópodos benéficos; $\mathrm{P}=$ vida media en la superficie de planta. Considerando estas propiedades para cada ingrediente activo, dosis y concentración del producto utilizado se calcula el valor del EIQ de campo que nos permite comparar distintos productos o sistemas de producción, como así también considerar este valor a la hora de decidir la aplicación de un determinado tratamiento fitosanitario.

Los sistemas de producción de hortalizas de hoja son intensivos y los productos, al ser comercializados en fresco, deben cumplir condiciones de inocuidad para los consumidores. Por ello el cumplimiento de los días de carencia y el uso de productos fitosanitarios de bajo impacto ambiental son dos criterios fundamentales a la hora de decidir la aplicación de un tratamiento químico. Considerando lo anteriormente expuesto se realizó una recopilación bibliográfica de los productos autorizados para el control de plagas en cultivos hortícolas de hojas a los fines de facilitar a los asesores técnicos una guía para la toma de decisiones en el momento de realizar una recomendación de control químico.

\section{Principios activos utilizados en cultivos de hoja}

Se recopilaron datos de insecticidas, acaricidas y fungicidas de bajo período de carencia (menor a 8 días) para cultivos hortícolas de hoja como acelga, espinaca, rúcula, perejil, lechuga, repollo, apio y achicoria. Los datos que se relevaron fueron: principios activos registrados por cultivo, concentraciones de los productos, plagas o enfermedades que controlan, dosis, período de carencia, grupos químicos, bandas toxicológicas, formulaciones de los productos, su respectiva clasificación según el mecanismo de acción y el componente ecológico del EIQ según la dosis recomendada y considerando un volumen de aplicación de 2431 ha $^{-1}$ (volumen obtenido de relevamiento propio). Posteriormente se confeccionaron dos tipos de tablas con los principios activos para cada cultivo, una con insecticidas y acaricidas; y la otra referida a fungicidas.

El relevamiento de los principios activos, concentraciones de los productos, plagas o enfermedades que controlan, dosis, período de carencia y grupos químicos fueron recopilados de la Resolución 608-2012 para cultivos menores del SENASA (SENASA., 2012), Guía de Productos Fitosanitarios (CASAFE, 2013) y LMR (Límite Máximo de Residuo) por principio activo y por cultivo (SENASA, 2015). La clasificación de mecanismos de acción se realizó según IRAC (Insecticide Resistance Action Committee) (IRAC, 2017) y FRAC (Fungicide Resistance Action Committee) (FRAC, 2017) para los insecticidas y fungicidas respectivamente. Para la clasificación toxicológica se consultó el listado actualizado de formulados autorizados por SENASA hasta abril de 2017 (SENASA, 2017). Los valores del componente ecológico del EIQ se calcularon empleando la dosis requerida para un caudal de 243 1/ha (valor de caudal por hectárea obtenido por relevamiento propio) a través del calculador online (Eshenaur et al., 2015). Con estos datos se construyeron tablas que permiten visualizar estos parámetros y compararlos a fin de decidir que producto recomendar para el control de alguna plaga.

Para la confección de las tablas se tuvieron presentes las plagas que afectan los cultivos de acelga, espinaca, rúcula, perejil, lechuga, repollo, apio y achicoria. En todos los casos se calculó el valor del componente ecológico del EIQ con las dosis aconsejadas en la bibliografía, aunque en algunas situaciones este coeficiente fue calculado con otros principios activos de la misma familia química ya que el recomendado no estaba en la lista (datos resaltados con asteriscos en las tablas indicadas abajo). Se registraron en total 30 principios activos de bajo período de carencia, de los cuales 15 principios activos son insecticidas o acaricidas (Tablas 1,2 y 3) y 15 fungicidas (Tablas 4, 5 y 6). Solo en el caso de los fungicidas para rúcula, se indicaron 2 principios activos (Carbendazim y Clorotalonil) con un período de carencia mayor a 8 días. Esto es así, porque no se hallaron principios activos con menor período de carencia.

Entre las características que debemos observar al elegir un principio activo están: la clasificación toxicológica y los modos de acción que están siendo promovidos por las organizaciones anti-resistencia. Esto permitirá elegir tratamientos de menor riesgo para el operario aplicador y que los mismos sean elegidos con una adecuada rotación de los distintos modos de acción con el objetivo de retrasar la aparición de organismos resistentes. 


\section{CONSIDERACIONES FINALES}

Los datos son orientativos y fueron recopilados de resoluciones y documentaciones oficiales. Al momento de realizar un tratamiento fitosanitario, éste debe ser recomendado por un Ingeniero Agrónomo habilitado para tal fin y que debiera considerar las posibles modificaciones de los valores. La utilización adecuada de esta información contribuirá a una producción de hortalizas inocuas para el consumo y con el menor impacto ambiental posible.

Tabla N¹: Insecticidas y acaricidas en espinaca, rúcula y perejil

\begin{tabular}{|c|c|c|c|c|c|c|c|c|c|c|}
\hline & $\begin{array}{l}\text { Principio } \\
\text { activo }\end{array}$ & $\begin{array}{l}\% \\
\text { (1) }\end{array}$ & Plaga & Dosis & $\begin{array}{l}\text { PC } \\
\text { (2) }\end{array}$ & $\begin{array}{l}\text { Grupo } \\
\text { Químico }\end{array}$ & $\begin{array}{l}\text { ClasTox } \\
(6)\end{array}$ & $\begin{array}{c}\text { Form } \\
(3)\end{array}$ & $\begin{array}{c}\text { Clas } \\
\text { MoA } \\
\text { IRAC } \\
\text { (4) }\end{array}$ & \begin{tabular}{|c|} 
Comp \\
Eco \\
ElQc \\
$(5)$ \\
\end{tabular} \\
\hline \multirow{7}{*}{ 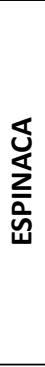 } & Abamectina & 1,8 & Arañuelas & $100 \mathrm{~cm}^{3} / \mathrm{hl}$ & 3 & Avermectina & II; III; IV & EC & 6 & 0,3 \\
\hline & Clofentezine & 50 & Arañuelas & $50 \mathrm{~cm}^{3} / \mathrm{hl}$ & 7 & Tetrazina & II & SC & $10 \mathrm{~A}$ & 3,4 \\
\hline & Spinosad & 48 & Trips & $15 \mathrm{~cm}^{3} / \mathrm{hl}$ & 5 & Spinosina & IV & SC & 5 & 0,5 \\
\hline & Lambdacialotrina & 5 & $\begin{array}{l}\text { Mosca Blanca; Trips; } \\
\text { Orugas; Pulgones }\end{array}$ & $125 \mathrm{~cm}^{3} / \mathrm{hl}$ & 3 & Piretroide & Ib; II; III & EC & $3 A$ & 1,4 \\
\hline & Imidacloprid & 35 & Mosca Blanca; Pulgones & $50 \mathrm{~cm}^{3} / \mathrm{hl}$ & 5 & Neonicotinoide & II; III & SC & $4 \mathrm{~A}$ & 3,4 \\
\hline & Acetamiprid & 20 & Mosca Blanca; Pulgones & $50 \mathrm{gr} / \mathrm{hl}$ & 3 & Neonicotinoide & II; III & SP & $4 \mathrm{~A}$ & 1,6 \\
\hline & Bifentrin & 10 & $\begin{array}{l}\text { Mosca Blanca; Trips; } \\
\text { Pulgones; Orugas }\end{array}$ & $30 \mathrm{~cm}^{3} / \mathrm{hl}$ & 3 & Piretroide & Ib; II & EC & $3 A$ & 0,7 \\
\hline \multirow{7}{*}{ 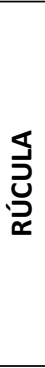 } & Abamectina & 1,8 & Arañuelas & $100 \mathrm{~cm}^{3} / \mathrm{hl}$ & 3 & Avermectina & II; III; IV & EC & 6 & 0,3 \\
\hline & Pirimicarb & 50 & Pulgones & $165 \mathrm{gr} / \mathrm{hl}$ & 7 & Carbamatos & II & WG & $1 \mathrm{~A}$ & 6,1 \\
\hline & Pyriproxifen & 10 & Mosca Blanca & $75 \mathrm{~cm}^{3} / \mathrm{hl}$ & 7 & Juvenoides & III; IV & EC & $7 C$ & 0,6 \\
\hline & Lambdacialotrina & 5 & $\begin{array}{l}\text { Mosca Blanca; Trips; } \\
\text { Orugas; Pulgones }\end{array}$ & $125 \mathrm{~cm}^{3} / \mathrm{hl}$ & 3 & Piretroide & Ib; II; III & EC & $3 \mathrm{~A}$ & 1,4 \\
\hline & Imidacloprid & 35 & Mosca Blanca; Pulgones & $50 \mathrm{~cm}^{3} / \mathrm{hl}$ & 5 & Neonicotinoide & II; III & SC & $4 \mathrm{~A}$ & 3,4 \\
\hline & Acetamiprid & 20 & Mosca Blanca; Pulgones & $50 \mathrm{gr} / \mathrm{hl}$ & 3 & Neonicotinoide & II; III & SP & $4 \mathrm{~A}$ & 1,6 \\
\hline & Bifentrin & 10 & $\begin{array}{c}\text { Mosca Blanca; Trips; } \\
\text { Pulgones; Orugas }\end{array}$ & $30 \mathrm{~cm}^{3} / \mathrm{hl}$ & 3 & Piretroide & Ib; II & EC & $3 A$ & 0,7 \\
\hline \multirow{4}{*}{ 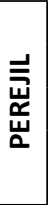 } & Tiametoxam & 25 & Mosca Blanca y Pulgones & $50 \mathrm{gr} / \mathrm{hl}$ & 3 & Neonicotinoide & IV & EG & $4 \mathrm{~A}$ & 2,1 \\
\hline & Spinosad & 48 & Trips & $15 \mathrm{~cm}^{3} / \mathrm{hl}$ & 3 & Spinosina & IV & SC & 5 & 0,5 \\
\hline & Formetanato & 50 & Trips & $100 \mathrm{gr} / \mathrm{hl}$ & 7 & Carbamato & II & SP & $1 \mathrm{~A}$ & 4,8 \\
\hline & Lambdacialotrina & 5 & $\begin{array}{c}\text { Gusanos cortadores; } \\
\text { Pulgones }\end{array}$ & $125 \mathrm{~cm}^{3} / \mathrm{hl}$ & 1 & Piretroide & Ib; II; III & EC & $3 A$ & 1,4 \\
\hline
\end{tabular}

Tabla N²: Insecticidas y acaricidas en repollo, apio y achicoria

\begin{tabular}{|c|c|c|c|c|c|c|c|c|c|c|}
\hline & Principio activo & $\begin{array}{l}\% \\
\text { (1) }\end{array}$ & Plaga & Dosis & $\begin{array}{l}P C \\
\text { (2) }\end{array}$ & $\begin{array}{c}\text { Grupo } \\
\text { Químico }\end{array}$ & $\begin{array}{l}\text { ClasTox } \\
\text { (6) }\end{array}$ & $\begin{array}{c}\text { Form } \\
(3)\end{array}$ & $\begin{array}{c}\text { Clas } \\
\text { MoA } \\
\text { IRAC } \\
(4) \\
\end{array}$ & $\begin{array}{c}\text { Comp } \\
\text { Eco } \\
\text { EIQc } \\
(5) \\
\end{array}$ \\
\hline \multirow{5}{*}{$\begin{array}{l}\text { 을 } \\
\text { 윰 } \\
\text { 또 }\end{array}$} & Deltametrina & 10 & Palomita de coles; Pulgón. & $10 \mathrm{~cm}^{3} / \mathrm{hl}$ & 3 & Piretroide & II; III; IV & EC & $3 A$ & 0,1 \\
\hline & \multirow{2}{*}{ Imidacloprid } & \multirow{2}{*}{35} & \multirow{2}{*}{ Pulgón. } & $30 \mathrm{~cm}^{3} / \mathrm{hl}$ & \multirow{2}{*}{7} & \multirow{2}{*}{ Neonicotinoide } & \multirow{2}{*}{ II ;III } & \multirow{2}{*}{ SC } & \multirow{2}{*}{$4 \mathrm{~A}$} & 2 \\
\hline & & & & $50 \mathrm{~cm}^{3} / \mathrm{hl}$ & & & & & & 3,4 \\
\hline & Pirimicarb & 50 & Pulgón & $500 \mathrm{gr} / \mathrm{ha}$ & 5 & Carbamatos & II & WG & $1 \mathrm{~A}$ & 7,6 \\
\hline & Flubendiamide & 48 & $\begin{array}{l}\text { Oruga cortadora (Agrotis } \\
\text { ipsylon); Isoca de las coles } \\
\text { (Plutella xylostella); Isoca } \\
\text { Medidora (Rachiplusia nu) }\end{array}$ & $20 \mathrm{~cm}^{3} / \mathrm{hl}$ & 3 & Diamidas & III & SC & 28 & 0,9 \\
\hline \multirow{4}{*}{$\frac{0}{2}$} & Abamectina & 1,8 & Arañuelas; Lyriomiza & $80 \mathrm{~cm}^{3} / \mathrm{hl}$ & 3 & Avermectina & II; III; IV & EC & 6 & 0,3 \\
\hline & Cartap & 45 & Liriomyza spp & $105 \mathrm{gr} / \mathrm{hl}$ & 7 & Tiocarbamato & II; IV & SP & 14 & 9 \\
\hline & & & \multirow{2}{*}{ Arañuelas } & $30 \mathrm{gr} / \mathrm{hl}$ & \multirow{2}{*}{7} & \multirow{2}{*}{$\begin{array}{l}\text { Triazolidina } \\
\text { carboxamida }\end{array}$} & \multirow{2}{*}{ IV } & \multirow{2}{*}{ WP } & \multirow{2}{*}{$10 \mathrm{~A}$} & 0,5 \\
\hline & Hexytiazox & 10 & & $50 \mathrm{gr} / \mathrm{hl}$ & & & & & & 0,8 \\
\hline \multirow{7}{*}{ 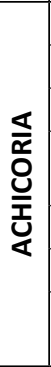 } & Abamectina & 1,8 & Arañuelas; Lyriomiza. & $100 \mathrm{~cm}^{3} / \mathrm{hl}$ & 3 & Avermectina & II; III; IV & EC & 6 & 0,3 \\
\hline & Formetanato & 50 & Trips & $100 \mathrm{gr} / \mathrm{hl}$ & 7 & Carbamato & II & SP & $1 \mathrm{~A}$ & 4,8 \\
\hline & Spinosad & 48 & Lyriomiza; Trips. & $15 \mathrm{~cm}^{3} / \mathrm{hl}$ & 5 & Spinosina & IV & SC & 5 & 0,5 \\
\hline & Lambdacialotrina & 5 & $\begin{array}{l}\text { Mosca blanca; Trips; } \\
\text { Pulgones }\end{array}$ & $125 \mathrm{~cm}^{3} / \mathrm{hl}$ & 3 & Piretroide & Ib; II; III & EC & $3 A$ & 1,4 \\
\hline & Imidacloprid & 35 & Mosca Blanca; Pulgones & $50 \mathrm{~cm}^{3} / \mathrm{hl}$ & 3 & Neonicotinoide & II; III & SC & $4 \mathrm{~A}$ & 3,4 \\
\hline & Acetamiprid & 20 & Mosca Blanca; Pulgones & $50 \mathrm{gr} / \mathrm{hl}$ & 3 & Neonicotinoide & II; III & SP & $4 \mathrm{~A}$ & 1,6 \\
\hline & Bifentrin & 10 & $\begin{array}{c}\text { Mosca Blanca; Trips; } \\
\text { Pulgones. }\end{array}$ & $30 \mathrm{~cm}^{3} / \mathrm{hl}$ & 3 & Piretroide & Ib; II & EC & $3 A$ & 0,7 \\
\hline
\end{tabular}


Tabla No 3: Insecticidas y acaricidas en acelga y lechuga

\begin{tabular}{|c|c|c|c|c|c|c|c|c|c|c|}
\hline & $\begin{array}{l}\text { Principio } \\
\text { activo }\end{array}$ & $\begin{array}{l}\% \\
\text { (1) }\end{array}$ & Plaga & Dosis & $\begin{array}{l}\mathrm{PC} \\
\text { (2) }\end{array}$ & $\begin{array}{l}\text { Grupo } \\
\text { Químico }\end{array}$ & $\begin{array}{l}\text { ClasTox } \\
(6)\end{array}$ & $\begin{array}{c}\text { Form } \\
\text { (3) }\end{array}$ & $\begin{array}{c}\text { Clas } \\
\text { MoA } \\
\text { IRAC } \\
\text { (4) }\end{array}$ & \begin{tabular}{|c|} 
Comp \\
Eco \\
EIQc \\
$(5)$ \\
\end{tabular} \\
\hline \multirow{9}{*}{ త্ّ } & Deltametrina & 10 & Bicho moro & $7 \mathrm{~cm}^{3} / \mathrm{hl}$ & 7 & Piretroide & II; III; IV & $\mathrm{EC}$ & $3 A$ & 0,1 \\
\hline & Abamectina & 1,8 & Arañuelas & $100 \mathrm{~cm}^{3} / \mathrm{hl}$ & 3 & Avermectina & II; III; IV & EC & 6 & 0,3 \\
\hline & Clofentezine & 50 & Arañuelas & $50 \mathrm{~cm}^{3} / \mathrm{hl}$ & 7 & Tetrazina & II & SC & $10 \mathrm{~A}$ & 3,4 \\
\hline & Spinosad & 48 & Lyriomiza. Trips. & $15 \mathrm{~cm}^{3} / \mathrm{hl}$ & 5 & Spinosina & IV & SC & 5 & 0,5 \\
\hline & Lambdacialotrina & 5 & $\begin{array}{l}\text { Mosca Blanca; Trips; } \\
\text { Orugas; Pulgones }\end{array}$ & $125 \mathrm{~cm}^{3} / \mathrm{hl}$ & 3 & Piretroide & Ib; II; III & EC & $3 A$ & 1,4 \\
\hline & Tiametoxam & 25 & $\begin{array}{c}\text { Mosca Blanca; Pulgones; } \\
\text { Trips }\end{array}$ & $50 \mathrm{gr} / \mathrm{hl}$ & 1 & Neonicotinoide & IV & EG & $4 \mathrm{~A}$ & 2,1 \\
\hline & Imidacloprid & 35 & $\begin{array}{c}\text { Lyriomiza; Mosca Blanca; } \\
\text { Pulgones }\end{array}$ & $50 \mathrm{~cm}^{3} / \mathrm{hl}$ & 3 & Neonicotinoide & II; III & SC & $4 \mathrm{~A}$ & 3,4 \\
\hline & Acetamiprid & 20 & Mosca Blanca; Pulgones & $50 \mathrm{gr} / \mathrm{hl}$ & 3 & Neonicotinoide & II; III & SP & $4 \mathrm{~A}$ & 1,6 \\
\hline & Bifentrin & 10 & $\begin{array}{c}\text { Mosca Blanca; Trips; } \\
\text { Pulgones; Orugas }\end{array}$ & $30 \mathrm{~cm}^{3} / \mathrm{hl}$ & 3 & Piretroide & Ib; II & $\mathrm{EC}$ & $3 A$ & 0,7 \\
\hline \multirow{9}{*}{ 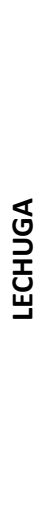 } & Abamectina & 1,8 & Arañuelas; Lyriomiza & $100 \mathrm{~cm}^{3} / \mathrm{hl}$ & 3 & Avermectina & II; III; IV & EC & 6 & 0,3 \\
\hline & Pirimicarb & 50 & Pulgones & $500 \mathrm{gr} / \mathrm{ha}$ & 3 & Carbamatos & II & WG & $1 \mathrm{~A}$ & 7,6 \\
\hline & Pyriproxifen & 10 & Mosca blanca & $75 \mathrm{~cm}^{3} / \mathrm{hl}$ & 3 & Juvenoides & III; IV & $E C$ & $7 C$ & 0,6 \\
\hline & Lambdacialotrina & 5 & $\begin{array}{l}\text { Mosca blanca; Trips; } \\
\text { Orugas; Pulgones }\end{array}$ & $125 \mathrm{~cm}^{3} / \mathrm{hl}$ & 3 & Piretroide & Ib; II; III & $\mathrm{EC}$ & $3 \mathrm{~A}$ & 1,4 \\
\hline & Imidacloprid & 35 & $\begin{array}{l}\text { Mosca blanca; Lyriomiza; } \\
\text { Pulgones }\end{array}$ & $50 \mathrm{~cm}^{3} / \mathrm{hl}$ & 7 & Neonicotinoide & II; III & SC & $4 \mathrm{~A}$ & 3,4 \\
\hline & Acetamiprid & 20 & Mosca blanca; Pulgones & $50 \mathrm{gr} / \mathrm{hl}$ & 3 & Neonicotinoide & II; III & SP & $4 \mathrm{~A}$ & 1,6 \\
\hline & Spinosad & 48 & Lyriomiza; Trips. & $15 \mathrm{~cm}^{3} / \mathrm{hl}$ & 5 & Spinosina & IV & SC & 5 & 0,5 \\
\hline & Bifentrin & 10 & $\begin{array}{l}\text { Mosca blanca; Trips; } \\
\text { Pulgones; Orugas }\end{array}$ & $30 \mathrm{~cm}^{3} / \mathrm{hl}$ & 3 & Piretroide & Ib; II & $\mathrm{EC}$ & $3 \mathrm{~A}$ & 0,7 \\
\hline & Flubendiamide & 48 & $\begin{array}{l}\text { Oruga cortadora (Agroti } \\
\text { sipsylon) }\end{array}$ & $20 \mathrm{~cm}^{3} / \mathrm{hl}$ & 3 & Diamidas & III & SC & 28 & 0,9 \\
\hline
\end{tabular}

Tabla No4: Fungicidas en repollo apio y achicoria.

\begin{tabular}{|c|c|c|c|c|c|c|c|c|c|c|}
\hline & $\begin{array}{l}\text { Principio } \\
\text { activo }\end{array}$ & $\begin{array}{l}\% \\
\text { (1) }\end{array}$ & Enfermedad & Dosis & $\begin{array}{l}\text { PC } \\
\text { (2) }\end{array}$ & $\begin{array}{l}\text { Grupo } \\
\text { Químico }\end{array}$ & $\begin{array}{l}\text { Clas Tox } \\
\text { (6) }\end{array}$ & $\begin{array}{c}\text { Form } \\
\text { (3) }\end{array}$ & $\begin{array}{c}\text { Clas } \\
\text { MoA } \\
\text { FRAC } \\
\text { (4) }\end{array}$ & \begin{tabular}{|c|} 
Comp \\
Eco \\
EIQc \\
$(5)$ \\
\end{tabular} \\
\hline \multirow{3}{*}{$\begin{array}{l}\text { 움 } \\
\text { 윰 } \\
\text { 똠 }\end{array}$} & Kasugamicina & 2 & $\begin{array}{l}\text { Podredumbre blanda } \\
\text { de las hortalizas }\end{array}$ & $250 \mathrm{~cm}^{3} / \mathrm{hl}$ & 1 & Antibiótico & IV & SL & D3 & \\
\hline & Carbendazim & 50 & $\begin{array}{l}\text { Podredumbre gris } \\
\text { (Botrytis cinerea) }\end{array}$ & $50 \mathrm{~cm} 3 / \mathrm{hl}$ & 7 & Bencimidazol & III; IV & SC & B1 & 4,5 \\
\hline & Mancozeb & 80 & $\begin{array}{c}\text { Mildiu de las } \\
\text { crucíferas }\end{array}$ & $200 \mathrm{gr} / \mathrm{hl}$ & 7 & Ditiocarbamato & II; III; IV & WP & $\begin{array}{c}\mathrm{M} \\
\text { (multisitio) }\end{array}$ & 16,9 \\
\hline \multirow{8}{*}{$\frac{0}{\frac{0}{\alpha}}$} & Azoxistrobina & 25 & $\begin{array}{l}\text { Viruela del apio } \\
\text { (Cercospora apii) } \\
\text { (Septoria apiicola) }\end{array}$ & $85 \mathrm{~cm}^{3} / \mathrm{hl}$ & 7 & Estrobilurina & II; III; IV & SC & C3 & 2,9 \\
\hline & Captan & 80 & $\begin{array}{l}\text { Viruela del apio } \\
\text { (Cercospora apii) }\end{array}$ & $150 \mathrm{~g} / \mathrm{hl}$ & 7 & Ftalimida & III; IV & WP & $\begin{array}{c}\mathrm{M} \\
\text { (multisitio) }\end{array}$ & 7,9 \\
\hline & \multirow{2}{*}{ Carbendazim } & \multirow{2}{*}{50} & \multirow{2}{*}{$\begin{array}{l}\text { Viruela del apio } \\
\text { (Cercospora apii) }\end{array}$} & $60 \mathrm{~cm} 3 / \mathrm{hl}$ & \multirow{2}{*}{7} & \multirow{2}{*}{ Bencimidazol } & \multirow{2}{*}{ III;IV } & \multirow{2}{*}{ SC } & \multirow{2}{*}{ B1 } & 5,4 \\
\hline & & & & $100 \mathrm{~cm} 3 / \mathrm{hl}$ & & & & & & 8,9 \\
\hline & Folpet & 80 & Viruela del apio & $160 \mathrm{gr} / \mathrm{hl}$ & 7 & Ftalimida & III & WP & $\begin{array}{c}\mathrm{M} \\
\text { (multisitio) }\end{array}$ & 21,4 \\
\hline & $\begin{array}{l}\text { Trifloxistrobin + } \\
\text { Tebuconazole }\end{array}$ & $\begin{array}{c}25+ \\
50\end{array}$ & $\begin{array}{l}\text { Viruela del apio } \\
\text { (Cercospora apii) }\end{array}$ & $40 \mathrm{gr} / \mathrm{hl}$ & 7 & $\begin{array}{c}\text { Estrobilurina + } \\
\text { Triazol }\end{array}$ & IV & WG & $\mathrm{C} 3+\mathrm{G} 1$ & 4,5 \\
\hline & \multirow{2}{*}{ Kasugamicina } & \multirow{2}{*}{2} & \multirow{2}{*}{ Viruela del apio } & 1,5 Its/ha & \multirow{2}{*}{1} & \multirow{2}{*}{ Antibiotico } & \multirow{2}{*}{ IV } & \multirow{2}{*}{ SL } & \multirow{2}{*}{ D3 } & \\
\hline & & & & 3 Its/ha & & & & & & \\
\hline \multirow{2}{*}{ 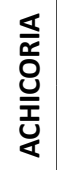 } & Azoxistrobina & 25 & $\begin{array}{l}\text { Oidio y Botrytis } \\
\text { cinerea }\end{array}$ & $125 \mathrm{~cm}^{3} / \mathrm{hl}$ & 7 & Estrobilurina & II ; III; IV & SC & $\mathrm{C} 3$ & 4,3 \\
\hline & Procimidone(*) & 50 & (Botrytis cinerea) & $100 \mathrm{~cm}^{3} / \mathrm{hl}$ & 7 & Dicarboximida & III & SC & E3 & $4,9 *$ \\
\hline
\end{tabular}


Tabla N5: Fungicidas en rúcula; perejil y lechuga

\begin{tabular}{|c|c|c|c|c|c|c|c|c|c|c|}
\hline & $\begin{array}{l}\text { Principio } \\
\text { activo }\end{array}$ & $\begin{array}{l}\% \\
\text { (1) }\end{array}$ & Enfermedad & Dosis & $\begin{array}{l}\text { PC } \\
\text { (2) }\end{array}$ & $\begin{array}{l}\text { Grupo } \\
\text { Químico }\end{array}$ & $\begin{array}{l}\text { Clas } \\
\text { Tox } \\
\text { (6) }\end{array}$ & $\begin{array}{l}\text { Form } \\
\text { (3) }\end{array}$ & $\begin{array}{c}\text { Clas } \\
\text { MoA } \\
\text { FRAC } \\
\text { (4) } \\
\end{array}$ & $\begin{array}{c}\text { Comp } \\
\text { Eco } \\
\text { EIQc } \\
(5)\end{array}$ \\
\hline \multirow{2}{*}{ 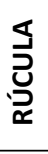 } & Carbendazim & 50 & $\begin{array}{l}\text { Botrytis; } \\
\text { Sclerotinia }\end{array}$ & $100 \mathrm{~cm}^{3} / \mathrm{hl}$ & 14 & Bencimidazol & III; IV & SC & B1 & 8,9 \\
\hline & Clorotalonil & 50 & Oídio & $300 \mathrm{~cm}^{3} / \mathrm{hl}$ & 14 & Cloronitrilo & III; IV & SC & $\begin{array}{c}\mathrm{M} \\
\text { (multisitio) }\end{array}$ & 25,3 \\
\hline \multirow{3}{*}{ 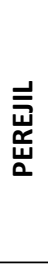 } & Azoxistrobina & 25 & \begin{tabular}{|c|} 
Peronospora, Oídio, \\
Phythium \\
y Cercospora sp.
\end{tabular} & $125 \mathrm{~cm}^{3} / \mathrm{hl}$ & 3 & Estrobilurina & II; III; & SC & C3 & 4,3 \\
\hline & Captan & 80 & Viruela del apio & $150 \mathrm{gr} / \mathrm{hl}$ & 7 & Ftalimida & III;IV & WP & $\begin{array}{c}\mathrm{M} \\
\text { (multisitio) }\end{array}$ & 7,9 \\
\hline & Penconazole $(* *)$ & 10 & $\begin{array}{c}\text { Oídio; } \\
\text { Cercospora sp. }\end{array}$ & $50 \mathrm{~cm}^{3} / \mathrm{hl}$ & 3 & Triazol & III & EC & G1 & $0,7 * *$ \\
\hline \multirow{6}{*}{ 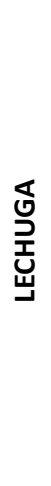 } & Azoxistrohina & \multirow{2}{*}{25} & \multirow{2}{*}{$\begin{array}{c}\text { Mildiu (Bremia } \\
\text { lactucae); Pythium } \\
\text { (Pythium spp.). }\end{array}$} & \multirow{2}{*}{$\begin{array}{l}125 \mathrm{~cm}^{3} / \mathrm{hl} \\
187,5 \mathrm{~cm}^{3} / \mathrm{hl}\end{array}$} & \multirow{2}{*}{7} & \multirow{2}{*}{ Estrobilurina } & \multirow{2}{*}{ II; III; } & \multirow{2}{*}{ SC } & \multirow{2}{*}{ C3 } & 4,3 \\
\hline & 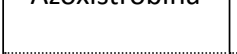 & & & & & & & & & 6,5 \\
\hline & Procimidone $(*)$ & 50 & $\begin{array}{c}\text { (Sclerotinias } \\
\text { clerotiorum) } \\
\text { (Botrytis cinerea) }\end{array}$ & $100 \mathrm{ml} / \mathrm{hl}$ & 7 & Dicarboximida & III & SC & E3 & $4,9 *$ \\
\hline & $\begin{array}{c}\text { Mancozeb + } \\
\text { Metalaxil - M }\end{array}$ & $64+4$ & $\begin{array}{l}\text { Hongos de suelo } \\
\text { (Pythium spp.) }\end{array}$ & $250 \mathrm{gr} / \mathrm{hl}$ & 7 & $\begin{array}{c}\text { Ditiocarbamato }+ \\
\text { Acilalanina }\end{array}$ & IV & WG & $\begin{array}{c}M \\
\text { (multisitio) } \\
+\mathrm{A} 1\end{array}$ & 17,7 \\
\hline & $\begin{array}{l}\text { Trifloxistrobin + } \\
\text { Tebuconazole }\end{array}$ & $25+50$ & $\begin{array}{c}\text { Moho gris (Botryti } \\
\text { ssp.) }\end{array}$ & $60 \mathrm{gr} / \mathrm{hl}$ & 5 & $\begin{array}{l}\text { Estrobilurina + } \\
\quad \text { Triazol }\end{array}$ & IV & WG & $\mathrm{C} 3+\mathrm{G} 1$ & 6,8 \\
\hline & $\begin{array}{l}\text { Fluopicolide + } \\
\text { Propamocarb }\end{array}$ & $6,25+62,5$ & $\begin{array}{l}\text { Mildiu (Bremia } \\
\text { lactucae) }\end{array}$ & $250 \mathrm{ml} / \mathrm{hl}$ & 7 & $\begin{array}{l}\text { Acylpicolide + } \\
\text { Carbamato }\end{array}$ & III & SC & $\mathrm{B} 5+\mathrm{F} 4$ & 16,6 \\
\hline
\end{tabular}

Tabla $N^{\mathbf{0}}$ 6: Fungicidas en acelga y espinaca

\begin{tabular}{|c|c|c|c|c|c|c|c|c|c|c|}
\hline & $\begin{array}{l}\text { Principio } \\
\text { activo }\end{array}$ & $\begin{array}{l}\% \\
\text { (1) }\end{array}$ & Enfermedad & Dosis & $\begin{array}{l}\text { PC } \\
\text { (2) }\end{array}$ & $\begin{array}{l}\text { Grupo } \\
\text { Químico }\end{array}$ & $\begin{array}{l}\text { Clas } \\
\text { Tox } \\
\text { (6) }\end{array}$ & $\begin{array}{c}\text { Form } \\
\text { (3) }\end{array}$ & $\begin{array}{c}\text { Clas } \\
\text { MoA } \\
\text { FRAC } \\
\text { (4) }\end{array}$ & $\begin{array}{c}\text { Comp } \\
\text { Eco } \\
\text { EIQc } \\
(5)\end{array}$ \\
\hline \multirow{7}{*}{ 㜽 } & \multirow{2}{*}{ Azoxistrobina } & \multirow{2}{*}{25} & \multirow{2}{*}{$\begin{array}{l}\text { Viruela de la acelga } \\
\text { (Cercospora beticola) }\end{array}$} & $125 \mathrm{~cm}^{3} / \mathrm{hl}$ & \multirow{2}{*}{8} & \multirow{2}{*}{ Estrobilurina } & \multirow{2}{*}{ II; III; } & \multirow{2}{*}{ SC } & \multirow{2}{*}{ C3 } & 4,3 \\
\hline & & & & $187,5 \mathrm{~cm}^{3} / \mathrm{hl}$ & & & & & & 6,5 \\
\hline & Tebuconazole & 25 & $\begin{array}{c}\text { Fusarium sp.; Alternaria } \\
\text { sp.; Cercospora sp. y } \\
\text { Oidio }\end{array}$ & $50 \mathrm{~cm}^{3} / \mathrm{hl}$ & 7 & Triazol & II; III; & SC & G1 & 1,8 \\
\hline & Procimidone(*) & 50 & Botrytis; Sclerotinia & $100 \mathrm{~cm}^{3} / \mathrm{hl}$ & 7 & Dicarboximida & III & SC & E3 & $4,9 *$ \\
\hline & Mancozeb & 80 & $\begin{array}{l}\text { Viruela de la acelga } \\
\text { (Cercospora beticola) }\end{array}$ & $200 \mathrm{gr} / \mathrm{hl}$ & 7 & Ditiocarbamato & II; III; & WP & $\begin{array}{c}\mathrm{M} \\
\text { (multisitio) } \\
\end{array}$ & 16,9 \\
\hline & $\begin{array}{l}\text { Trifloxistrobin + } \\
\text { Tebuconazole }\end{array}$ & $25+50$ & $\begin{array}{l}\text { Viruela de la acelga } \\
\text { (Cercospora beticola) }\end{array}$ & $40 \mathrm{gr} / \mathrm{hl}$ & 7 & $\begin{array}{c}\text { Estrobilurina + } \\
\text { Triazol }\end{array}$ & IV & WG & $\mathrm{C} 3+\mathrm{G} 1$ & 4,5 \\
\hline & Kasugamicina & 2 & Viruela de la acelga & $250 \mathrm{~cm}^{3} / \mathrm{hl}$ & 1 & Antibiótico & IV & SL & D3 & \\
\hline \multirow{3}{*}{ 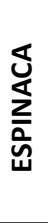 } & Azoxistrobina & 25 & $\begin{array}{c}\text { Mildiu de la espinaca } \\
\text { (Peronospora spinaceae) }\end{array}$ & $187,5 \mathrm{~cm}^{3} / \mathrm{hl}$ & 8 & Estrobilurina & $\begin{array}{l}\text { II; III; } \\
\text { IV }\end{array}$ & SC & C3 & 6,5 \\
\hline & Procimidone(*) & 50 & $\begin{array}{l}\text { Botrytis cinerea; } \\
\text { Sclerotinia sp }\end{array}$ & $100 \mathrm{~cm}^{3} / \mathrm{hl}$ & 7 & Dicarboximida & III & SC & E3 & $4,9 *$ \\
\hline & Mancozeb & 80 & $\begin{array}{c}\text { Mildiu de la espinaca } \\
\text { (Peronospora spinaceae) }\end{array}$ & $200 \mathrm{gr} / \mathrm{hl}$ & 7 & Ditiocarbamato & $\begin{array}{l}\text { II; III; } \\
\text { IV }\end{array}$ & WP & $\begin{array}{c}\mathrm{M} \\
\text { (multisitio) }\end{array}$ & 16,9 \\
\hline
\end{tabular}

Referencias de tablas:

- (1) Concentración del formulado.

- (2) Período de carencia: usualmente expresado en días, que deben transcurrir entre la última aplicación de un producto agroquímico y la cosecha.

- (3) Tipo de formulación.

- (4) Código de clasificación en función al Modo de Acción según el IRAC o el FRAC.

- (5) Componentes Ecológico del EIQ campo según la dosis para 243 1/hectárea.

- (6) Clasificación Toxicológica.

- $\quad$ *) Números de EIQ calculados con los valores para Iprodione.

- (**) Números de EIQ calculados con los valores para Tebuconazole. 


\section{BIBLIOGRAFÍA}

Arregui, M.C.; Sánchez, D.E.; Grenón, D.A. 2009. Tablero de comando sobre riesgo de contaminación ambiental por plaguicidas. Memorias. XXXVIII Jornadas Argentinas de Informática. Mar del Plata, BA, 24.

Arregui, M.C.; Grenón, D.; Sánchez, D.; Ghione, J. 2013. Evaluación del riesgo de impacto ambiental de plaguicidas en cultivos anuales del centro de Santa Fé. Fave. Sección ciencias agrarias, 12(1), 13-20.

CASAFE. 2013. Guía de Productos Fitosanitarios CASAFE 2013-2015. 16 a ed. Buenos Aires. 1185.

CASAFE. 2015. Guía de Productos Fitosanitarios CASAFE 2015-2017. 17 a ed. Buenos Aires. 1200.

Eshenaur, B.; Grant, J.; Kovach, J.; Petzoldt, C.; Degni, J.; Tette, J. 2015. Environmental Impact Quotient: "A Method to Measure the Environmental Impact of Pesticides.” New York State Integrated Pest Management Program, Cornell Cooperative Extension, Cornell University. Recuperado de: https://nysipm.cornell.edu/eiq/calculator-field-use-eiq.

FRAC. 2017. Información sobre clasificación de Modos de Acción de los Fungicidas. Obtenido de http:// www.frac.info/publications/downloads.

Goites, E. 2008. Manual de cultivos para la huerta orgánica familiar. Edición literaria a cargo de Janine Schonwald. - $1^{\text {era }}$ ed. - Buenos Aires - INTA. 136.

IRAC. 2017. Información sobre clasificación de Modos de Acción de los Insecticidas. Obtenido de: http://www. irac-online.org/documents/moa-classification/?ext=pdf
Kovach, J.; Petzoldt, C.; Degni, J.; Tette, J. 1992. A method to measure the environmental impact of pesticides. New York's Food and Life Sciences Bulletin 139:1-8.

Pacheco RM, Barbona EI. 2017. Manual de uso seguro y responsable de agroquímicos en cultivos frutihortícolas 1a ed. - Bella Vista, Corrientes. Ediciones INTA.

Reyes, G.; Chaparro-Giraldo, A.; Ávila, K. 2010. Efecto ambiental de agroquímicos y maquinaria agrícola en cultivos transgénicos y convencionales de algodón”. Rev. Colomb. Biotecnol. Vol XII N $2.151-162$.

SENASA. 2012. Resolución 608-2012 para cultivos menores del SENASA. Obtenido de: http://www. senasa.gob.ar/resolucion-6082012>. Ultimo acceso 01/09/2017 a las 19:35 hs.

SENASA. 2015. Listado actualizado de LMR por activo y por cultivo de Mayo del 2015. Obtenido de: http:// www.senasa.gob.ar/informacion/prod-vet-fito-y-fertilizantes/prod-fitosanitarios-y-fertili/registro-nacionalde-terapeutica-vegetal. Último acceso 10/06/2017 a las 11:50 hs.

SENASA. 2017. Listado actualizado de Formulados de Abril del 2017. Obtenido de: http://www.senasa.gob. ar/informacion/prod-vet-fito-y-fertilizantes/prod-fitosanitarios-y-fertili/registro-nacional-de-terapeuticavegetal. Último acceso 10/06/2017 a las 11:57 hs. 